TITLE:

\title{
Monotonic Damping in Nanoscopic Hydration Experiments
}

\section{$\operatorname{AUTHOR}(\mathrm{S})$ :}

Labuda, Aleksander; Kobayashi, Kei; Suzuki, Kazuhiro; Yamada, Hirofumi; Gruetter, Peter

\section{CITATION:}

Labuda, Aleksander ...[et al]. Monotonic Damping in Nanoscopic

Hydration Experiments. Physical Review Letters 2013, 110(6): 066102.

ISSUE DATE:

2013-02-07

URL:

http://hdl.handle.net/2433/187998

RIGHT:

(C) 2013 American Physical Society 


\title{
Monotonic Damping in Nanoscopic Hydration Experiments
}

\author{
Aleksander Labuda, ${ }^{1,2}$ Kei Kobayashi, ${ }^{3}$ Kazuhiro Suzuki, ${ }^{3}$ Hirofumi Yamada, ${ }^{3}$ and Peter Grütter ${ }^{2}$ \\ ${ }^{1}$ Asylum Research an Oxford Instruments Company, Santa Barbara, California 93117, USA \\ ${ }^{2}$ Department of Physics, McGill University, Montreal, Quebec H3A 2T8, Canada \\ ${ }^{3}$ Department of Electronic Science and Engineering, Kyoto University, Kyoto 615-8510, Japan
}

(Received 29 September 2012; published 7 February 2013)

\begin{abstract}
We present the first accurate damping profiles acquired in atomic-resolution hydration force spectroscopy, revealing a monotonic damping profile at the nanoscopic tip apex. Atomic resolution is confirmed by the observed inhomogeneity caused by random substitution of Si by $\mathrm{Al}$ on the mica surface, and two distinct damping regimes are identified. Damping only appears above our detection limit of $1 \mathrm{nNs} / \mathrm{m}$ once the tip interacts with the second hydration layer, and an onset of strong damping above $100 \mathrm{nNs} / \mathrm{m}$ appears upon direct interaction with the adsorbed layer and first hydration layer. These results are compared to various simulations to interpret the damping signals and determine the tip-sample distance.
\end{abstract}

The peculiar nanoscale structure of bulk water that explains its anomalous macroscopic properties, such as the nonmonotonic temperature dependence of density, specific heat, and compressibility, is a topic of heated debate. Naturally, if the structure of pure bulk water itself is controversial, the hydration structures around complex proteins are even further from being understood. Yet, these hydration structures govern the biomolecular interactions of all living organisms. Molecular dynamic simulations $[1,2]$ shed light on this problem, but validation with experimental data is a necessary step in the process. The construction of more accurate models describing the dynamics and interaction potentials of water molecules are best validated by comparison with well-understood systems, rather than complex molecules. The mica surface represents a good candidate sample as its structure has been well characterized, and it can be cleanly and reliably prepared in a laboratory setting. Furthermore, the atomicscale texture of the mica surface templates an intricate, yet repeatable, three-dimensional distribution of water molecules $[3,4]$.

Atomic force microscopy has become a prevailing technique for exploring the mechanical properties of water near solid surfaces. Although the water-mica interface has been studied by many experimental methods, such as x-ray reflectivity [5] and surface force apparatus [6], atomic force microscopy (AFM) carries the advantage of threedimensional atomic-scale resolution, such as recently demonstrated by Fukuma et al. [7] and Kobayashi et al. [8]. Both studies used frequency modulation (FM) AFM to probe the distribution of water molecules on an atomistic level via direct force imaging by employing sharp tips. In this work, we extend their site-specific force spectroscopy investigations by analyzing the damping information made possible by photothermal excitation [9] used to excite the cantilever in our experiment. Our results provide complementary information to strengthen our understanding of dynamical properties of water molecules in different hydration layers.

Surface force apparatus studies suggest that the fluidity of water remains high near solids because of the suppression of hydrogen bond networks related to its solidlike behavior, whereas organic solvents see an increase in viscosity near solid interfaces due to the reduction in translation degrees of freedom [6]. Such studies have been complemented by AFM experiments which suggest that solidlike behavior of water can arise in certain dynamical conditions [10], thus far unattainable using a surface force apparatus. These recent results resolved a long-standing controversy that afflicted studies of interfacial water viscosity [11]. We extend this picture down to atomicresolution hydration experiments where experiments are performed in thermodynamic equilibrium and the damping profile is monotonic with respect to the surface distance.

Controversies linger regarding the existence of oscillatory versus monotonic damping profiles in squeeze-out experiments, especially in the field of nonpolar confined fluids [12-16]. The same consideration puts at variance the oscillatory damping profile at the mica interface [10] with respect to the monotonic damping profile we report in this Letter. These two types of experiments will be distinguished in this work to demonstrate that their results are in fact not contradictory.

The interpretation of dissipation measurements using FM-AFM in liquids [9], air [17], and vacuum [18] all require the knowledge of the transfer function of the excitation system $\mathcal{X}$ to prevent instrumental artifacts. The situation in liquids is the most intricate as the transfer function of the detection system $\mathcal{D}$ and the frequency dependence of viscous damping $\gamma(\omega)$ also affect the measurement. All these considerations make the drive amplitude (used to maintain constant cantilever oscillation amplitude) a frequency-dependent quantity, even in purely conservative interactions. Let the dimensionless 
calibration factor $(\omega)$ (where $ネ$ is the Japanese katakana symbol pronounced [ne]) represent the frequency dependence of the normalized drive amplitude $\Lambda$, defined as

$$
\text { ネ }(\omega)=\left|\frac{\sin \left(\theta_{\mathcal{C} s}-\Delta \theta_{\chi_{D}}\right)}{\sin \left(\theta_{\mathcal{C}}\right)}\right|^{-1}\left|\frac{\chi}{\chi_{s}}\right|^{-1}\left|\frac{\mathcal{D}}{\mathcal{D}_{s}}\right|^{-1}\left(\frac{\omega}{\omega_{s}}\right),
$$

where $s$ denotes quantities measured at the start of the experiment, $\theta_{\mathcal{C} s}$ is the phase of the cantilever, and $\Delta \theta_{\chi \mathcal{D}}$ is the frequency-dependent phase change in the excitation and detection systems. Before proceeding with the extraction of damping from $\Lambda$, any expected change in $\Lambda$ due to frequency dependence $ネ$ should be divided out, as in $\Lambda / ネ$. This calibration procedure is summarized by

$$
\gamma_{i}=\gamma_{s}\left[\frac{\Lambda(\omega)}{\hbar(\omega)}-\frac{\gamma(\omega)}{\gamma_{s}}\right]
$$

which is explained in detail in Ref. [9]. This equation was used to extract a monotonic damping profile from the oscillatory drive signal in Fig. 1 (See Supplemental Material for details [19]).
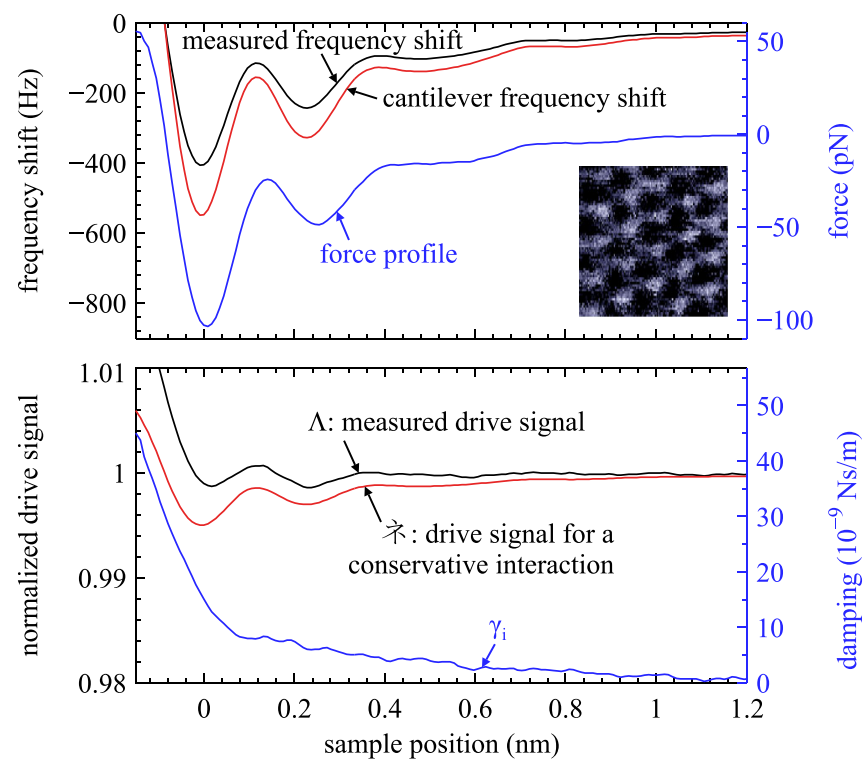

FIG. 1 (color online). These data are the result of averaging 8320 approach curves in water on mica $(0.25 \mathrm{M} \mathrm{KCl})$. They were acquired as a 3D image and averaged. The inset shows an $x y$ cross section of the frequency shift map at a constant height, arbitrarily assigned $z=0 \mathrm{~nm}$ for now. The PPP-NCH cantilever had $f_{0}=128.8 \mathrm{kHz}, Q=7.7, k=23.7 \mathrm{~N} / \mathrm{m}, A=0.16 \mathrm{~nm}$. (Top) The cantilever frequency shift was determined from the measured frequency shift by the theory in Ref. [9]. Then, a force deconvolution method was used to extract the force profile [20]. (Bottom) Equation (2) was used to extract $\gamma_{i}$ from the measured $\Lambda$ and the calculated $ネ$, while $\gamma(\omega)$ was calculated using Sader hydrodynamic theory [21]. More experimental details are available in Ref. [9].
The atomic-scale lateral resolution seen in the inset of Fig. 1 suggests that the interaction force is dominated by no more than a few atoms. Such a small tip apex results in small dissipative losses. The recovered damping on the order of $\sim 10^{-8} \mathrm{Ns} / \mathrm{m}$ is 5 orders of magnitude smaller than observed in a recent study of the dynamic solidification of confined water films [10] performed with a $\sim 100 \mathrm{~nm}$ tip radius. Before proceeding, these two types of AFM experiments need to be clearly distinguished; they are illustrated in Fig. 2.

Mesoscopic liquid confinement experiments study the rheological properties of water films trapped in between a blunt tip and a flat sample, using the framework of continuum hydrodynamics. At distances below $2 \mathrm{~nm}$, oscillatory forces are observed as they "rupture" individual water layers by displacing thousands of water molecules in unison, laterally to the surface. Such a thermodynamically irreversible process costs a large amount of energy and leads to large oscillatory damping and solidlike behavior in certain dynamical conditions [10], and depends on surface roughness [22]. These types of studies probe large contact areas as they relate to the mesoscopic properties of water films that are relevant to nanotribology and nanofluidics [23], for example.

In a nanoscopic hydration experiment, the tip-sample interaction involves a countable number of water molecules, whose dynamics and steric arrangements around single atoms are being probed. Sharp tip geometries allow the study of site-specific force profiles above atomically resolved surface atoms. The results are relevant for comparison to molecular dynamic simulations, with the goal of extending our understanding of water molecules at the nanoscopic scale. Such experiments are crucial for the understanding of biomolecular interactions, for example, which involve a small number of water molecules.

While our tip apex moves at around $100 \mu \mathrm{m} / \mathrm{s}$ with an oscillation period of $8 \mu \mathrm{s}$, water molecules move at an average speed of $600 \mathrm{~m} / \mathrm{s}$, collide with neighboring molecules $10^{8}$ times, and travel an average distance of $140 \mathrm{~nm}$ per cantilever oscillation cycle [24]. These fast dynamics imply that hydration layers displaced by a slowly

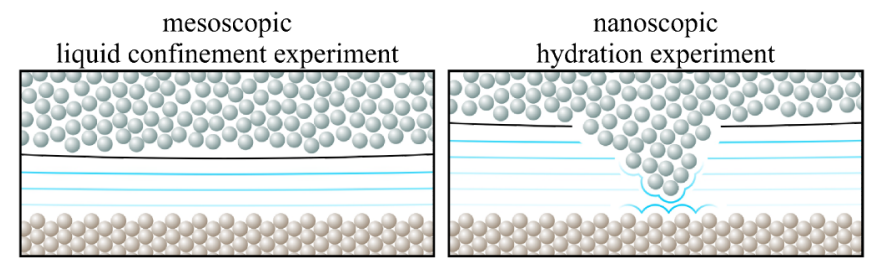

FIG. 2 (color online). In a mesoscopic liquid confinement experiment, continuum fluid dynamics are used to model the rheological properties of a water film confined between a tip (upper) and a surface (lower). The tip is characterized by its radius. In a nanoscopic hydration experiment, the dynamics of individual water molecules around the tip apex are being measured. 
approaching tip are continuously restored upon retraction of the tip - the process is quasistatic. These relatively fast relaxation times $(\sim$ ps) suggest that a nanoscopic hydration experiment should be highly adiabatic. However, we are aware that our experiment is not completely adiabatic even before approaching the surface, as $35 \mathrm{fW}$ of power are necessary to maintain the cantilever at constant amplitude. This power is dissipated into the bulk liquid by viscous loss across the full surface area of the cantilever and tip. The contribution from our nanoscopic tip apex to this nonadiabatic process is on the order of a yoctoWatt (yocto: $10^{-24}$ ), assuming a tip area of $\sim 1 \mathrm{~nm}^{2}$. Because of the oscillatory density profile of water molecules near a surface, one would then expect an oscillatory dissipation profile on the order of yoctoWatts (corresponding to a damping profile on the order of $10^{-15} \mathrm{Ns} / \mathrm{m}$ ).

Our detection threshold is $\sim 10^{-9} \mathrm{Ns} / \mathrm{m}$. A tremendous change in dynamical properties of water molecules would be necessary to increase the damping from $10^{-15} \mathrm{Ns} / \mathrm{m}$ to a detectable level. Molecular dynamics simulations predict very modest changes in the translational and rotational dynamics of water [25] until confinement separations of about $0.6 \mathrm{~nm}$ are reached [2]. Although the presence of $\mathrm{K}^{+}$ and $\mathrm{Cl}^{-}$ions retard the diffusion of water molecules due to ion hydration, this diffusion is reduced by at most 3 orders of magnitude under confinement relative to its bulk value [1]. Recently, Watkins and Shluger proposed that the interaction in a nanoscopic AFM hydration experiment can be broken down into "direct" and "water mediated" interactions with the surface [26]. Direct interactions are similar to the short-range interactions that occur in UHV environments, while water mediated interactions have an oscillatory component which can extend up to $\sim 1 \mathrm{~nm}$ from the surface. Neither of these models, nor the previously mentioned theoretical predictions, can account for the monotonic increase in damping that extends beyond $2 \mathrm{~nm}$ from the surface in our experiment.

The fact that our tip apex is tethered to an overhanging mesoscopic tip structure cannot be ignored, as suggested by Fig. 2. Squeeze film damping of the mesoscopic tip structure can cause a noticeable rise in dissipation in a nanoscopic experiment, which can be approximated as

$$
\gamma_{\mathrm{film}} \approx 6 \pi \eta R^{2} /\left(h+h_{0}\right)
$$

where $\eta$ is the viscosity, $R$ is the radius of the mesoscopic tip, $h$ is the height of the nanoscopic tip above the surface, and $h_{0}$ is the difference between the mesoscopic and the nanoscopic tip heights. Figure 3(a) shows the result of fitting the measured dissipation profile with a squeeze film damping contribution. Arbitrarily setting $h_{0}=2 \mathrm{~nm}$ returns a tip radius of $50 \mathrm{~nm}$ - on the order of what is expected.

As shown in Fig. 3(b), this mesoscopic squeeze film damping component was removed because it does not relate to the nanoscopic tip apex that is responsible for
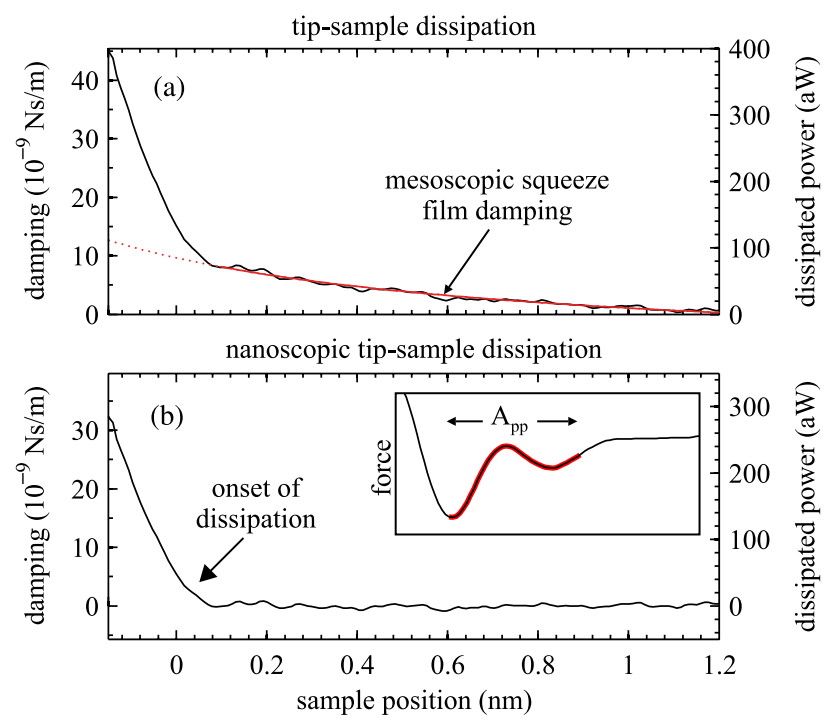

FIG. 3 (color online). (a) Tip-sample dissipation contains a slowly increasing background due to squeeze film damping (shown by fit) caused by an overhanging mesoscopic tip, on the order of what is expected from a typical AFM tip. (b) Removing this instrumental artifact isolates the nanoscopic dissipation profile, which shows a sudden onset of dissipation at $z \sim 0 \mathrm{~nm}$. This corresponds to the distance at which the tip encounters the strongest attractive force with the hydration layer, at the lowest point of its oscillation, as described in the text and illustrated in the inset along with the peak-to-peak amplitude of the oscillating cantilever.

atomic contrast. Now, a sudden onset of dissipation is observed near $z=0 \mathrm{~nm}$. The short range of the dissipation profile is indicative that it relates to interactions involving only the tip apex. The sudden onset of dissipation coincides with the situation where the tip apex encounters the most attractive force at the bottom of its oscillation cycle, as shown in the inset. The attractive minimum force corresponds to a situation where the second hydration layer tries to hydrate the tip and the sample simultaneously $[3,8]$.

Repeated deliberate tip crashes led to the atomic resolution images in Fig. 1. Upon tip crash, a fresh silicon surface was revealed and quickly oxidized due to the presence of water. It is therefore reasonable to assume that the tip of the cantilever is composed of silica. Although both surfaces are hydrophilic, the hydration characteristics of silica are qualitatively very different from mica: the water molecules have no long-range lateral order near silica [3]. Consequently, on the long time scales of an AFM experiment, the water distribution amounts to a probabilistic blur around the apex of the tip with no specific lateral features. This condition enables the cantilever tip to image the hydration structures of mica which dominate the measured lateral contrast.

The periodicity of the observed structure matches the pseudohexagonal lattice of the underlying mica. However, it is unclear a priori whether the mica surface or a 
hydration layer is being imaged. Recent Monte Carlo simulations [3] and molecular dynamic simulations [2] suggest that the lateral distribution of water molecules exhibits long-range order up to the third hydration layer, above which water displays mostly bulklike properties. Both the adsorbed and first hydration layers share a considerable portion of their hydrogen bonds with the highly structured mica surface [3]; they are only $0.1 \mathrm{~nm}$ apart. We will consider them together as the "adsorbed-first" layer in the following discussion, where the absolute tip-sample distance is determined by analyzing additional hydration maps.

In Fig. 4, two additional hydration maps are displayed alongside of the data from Fig. 1 which is now labeled hydration map $A$. In hydration map $B$, the tip successfully displaced an underlying hydration layer, as can be deduced from the force profile. The three cross sections show that lateral long-range order in the distribution of water molecules was observed in the frequency shift channel at three distinct heights. This information suggests that the onset of dissipation, observed in both hydration maps $A$ and $B$, occurs at the same distance where the interaction between the tip and the second hydration layer is most attractive. Furthermore, another regime of strong dissipation appears once the tip attractively interacts with the adsorbed-first hydration layer on the mica. This dissipation profile is the sum of a short-range direct interaction and a long-range water mediated interaction. Although we do not observe an oscillatory dissipation component as one might expect from a water mediated interaction, its existence below our detection threshold cannot be excluded.

In hydration map $C$, the tip approached the mica up to a repulsive interaction, providing us with a more direct measure of the tip-sample distance. Interestingly, a minor tip change near the end of the scan largely affected the force profile shape around the adsorbed-first hydration layer. A similar effect occurred in map $B$ (not shown). Although these tip changes greatly affected the imaging quality and force profiles, we refer to them as "minor" because they modified the tip-sample distance by no more than $30 \mathrm{pm}$ in both cases. Recent molecular dynamics simulations [26] demonstrated how slight changes in tip geometry can have large effects on the observed force contrast, which we directly observe here.

The cross section taken just above the adsorbed-first layer in map $C$ shows detail which could not be readily distinguished in the cross section of the second hydration layer, as indicated by the dotted circles. These features are reportedly caused by local electrostatic variations due to the random substitution of $\mathrm{Si}$ by $\mathrm{Al}$ on the mica surface [27], and possibly by the presence potassium ions [28]. Unfortunately, hydration map $C$ was acquired after switching from photothermal to piezoacoustic excitation of the cantilever, rendering the measurement of a dissipation profile impossible [9].
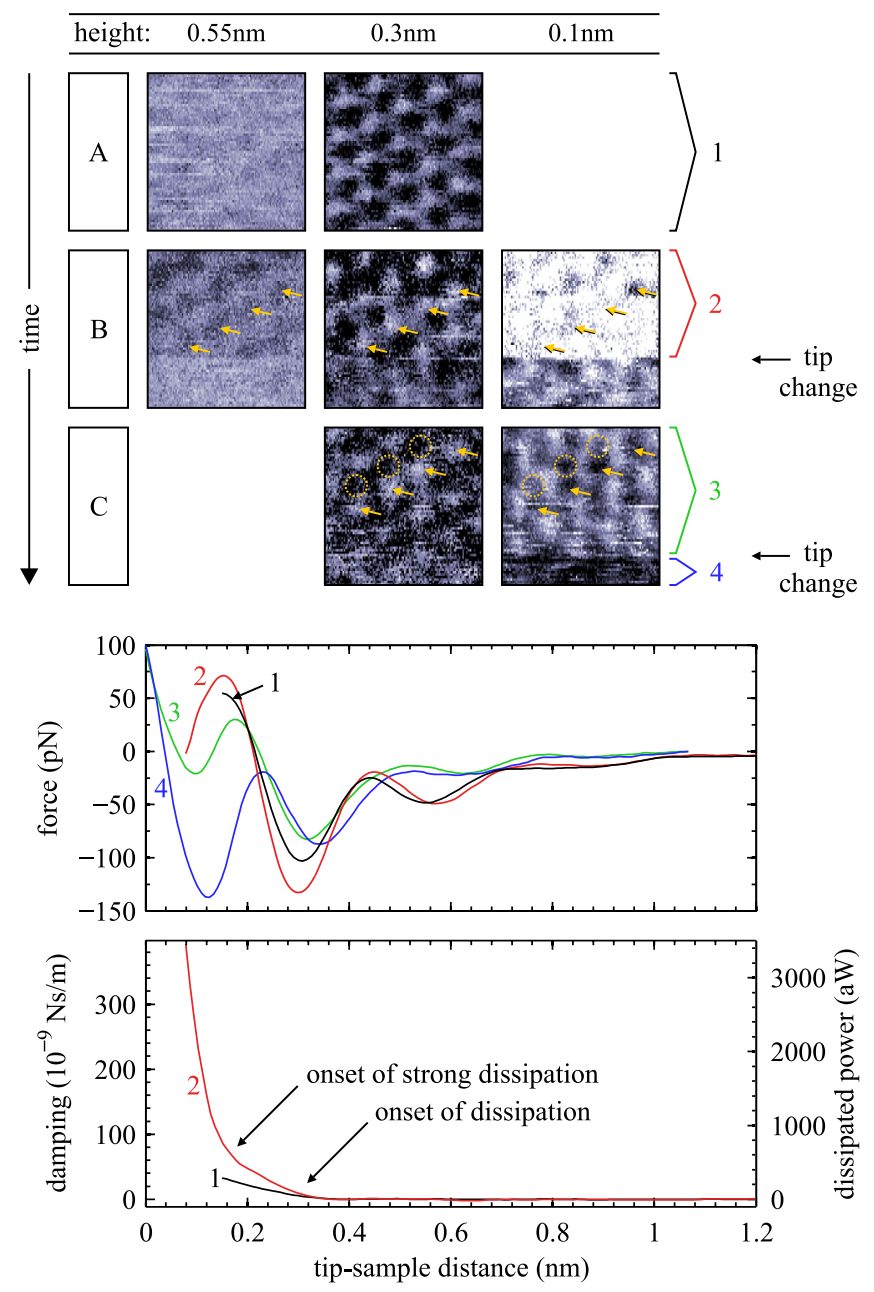

FIG. 4 (color online). The frequency shift hydration map from Fig. 1, labeled $A$, is shown along with two other hydration maps $(B$ and $C$ ), all acquired within one hour. The $x y$ cross sections were taken at different tip heights. The second hydration layer was displaced by the tip in data $B$, allowing it to interact with the adsorbed-first layer. The arrows aid the eye for observing the contrast inversion between adjacent images. In hydration map $C$, the tip reached a strong repulsive interaction. The circles show areas where local variations in the mica surface can be seen. Force spectroscopy corresponding to the images are plotted below.

Recent MD simulations [1] have specifically addressed the role of $\mathrm{K}^{+}$and $\mathrm{Cl}^{-}$ions in the hydration forces between mica sheets, in $1 \mathrm{M} \mathrm{KCl}$. The chloride atoms remain far from the surface (a few hydration layers) and therefore play little role in the hydration structures near the mica surface where atomic contrast is observed. In the same MD study, a damping mechanism was attributed to hydration profile hysteresis: the "forced adsorption" of $\mathrm{K}^{+}$ ions under extreme confinement and the subsequent "slow desorption" of the ions upon retraction. Importantly, although the simulated hydration force profiles are oscillatory, the observed hysteresis between approach and 
retraction curves was monotonic with tip-sample distance. These hysteretic ion dynamics represent another plausible damping mechanism to explain the monotonic damping we measured in our experiment.

In conclusion, our unprecedented instrumental characterization and new FM-AFM theory allowed us to unequivocally recover a monotonic damping profile in atomic-resolution hydration experiments at the mica surface. The onset of dissipation $>1 \mathrm{nNs} / \mathrm{m}$ upon interaction with the adsorbed-first hydration layer is attributed to large changes in rotational and translational dynamics of water molecules and/or hysteretic behavior of $\mathrm{K}^{+}$ions at the mica surface during approach and retraction of the AFM tip. The onset of strong dissipation $>100 \mathrm{nNs} / \mathrm{m}$ occurs upon direct interaction with the mica surface and was shown to result in unstable tip configurations. Importantly, we have distinguished between traditional liquid confinement experiments that observe large oscillatory damping profiles, and the nanoscopic hydration structure studies reported here that result in monotonic damping profiles.

Our methodology can be used for the determination of tip-sample distance which is necessary for direct comparison to simulations, and to provide complementary information regarding the physical properties of water molecules near solid surfaces. Measuring the site dependence of damping profiles is the next step necessary for quantifying and discriminating between the different proposed sources of dissipation. However, as can be deduced from a recent study [29], the damping signal in Fig. 3 is limited by the inherent force noise of the cantilever. Sitedependent studies of damping at the solid-liquid interface are expected to only be possible using smaller cantilevers, which have lower intrinsic force noise, and by direct excitation methods such as the photothermal method presented in this Letter.

The authors acknowledge valuable discussions with D. Kiracofe and software developed by N. Oyabu. Funding by FQRNT for this collaboration is greatly appreciated.

[1] Y. Leng, Langmuir 28, 5339 (2012).

[2] Y. Leng and P. T. Cummings, J. Chem. Phys. 124, 074711 (2006).

[3] A. Malani, K. G. Ayappa, and S. Murad, J. Phys. Chem. B 113, 13825 (2009).
[4] J. Wang, A. G. Kalinichev, R. J. Kirkpatrick, and R.T. Cygan, J. Phys. Chem. B 109, 15893 (2005).

[5] L. Cheng, P. Fenter, K. L. Nagy, M. L. Schlegel, and N. C. Sturchio, Phys. Rev. Lett. 87, 1 (2001).

[6] U. Raviv, P. Laurat, and J. Klein, Nature (London) 413, 51 (2001).

[7] T. Fukuma, Y. Ueda, S. Yoshioka, and H. Asakawa, Phys. Rev. Lett. 104, 016101 (2010).

[8] K. Kobayashi, N. Oyabu, K. Kimura, S. Ido, K. Suzuki, T. Imai, K. Tagami, M. Tsukada, and H. Yamada, (to be published).

[9] A. Labuda, K. Kobayashi, D. Kiracofe, K. Suzuki, P. H. Grütter, and H. Yamada, AIP Adv. 1, 022136 (2011).

[10] S. Khan, G. Matei, S. Patil, and P. Hoffmann, Phys. Rev. Lett. 105, 106101 (2010).

[11] S. Granick, S. Bae, S. Kumar, and C. Yu, Physics 3, 73 (2010).

[12] G. B. Kaggwa, J. I. Kilpatrick, J. E. Sader, and S. P. Jarvis, Appl. Phys. Lett. 93, 011909 (2008).

[13] S. J. O'Shea, Phys. Rev. Lett. 97, 179601 (2006).

[14] A. Maali, T. Cohen-Bouhacina, G. Couturier, and J.-P. Aimé, Phys. Rev. Lett. 96, 086105 (2006).

[15] S. D. Beer, W. K. D. Otter, D. V. D. Ende, W. J. Briels, and F. Mugele, Europhys. Lett. 97, 46001 (2012).

[16] L. Bureau, Phys. Rev. Lett. 104, 218302 (2010).

[17] R. Proksch and S. V. Kalinin, Nanotechnology 21, 455705 (2010).

[18] A. Labuda, Y. Miyahara, L. Cockins, and P.H. Grütter, Phys. Rev. B 84, 125433 (2011).

[19] See Supplemental Material at http://link.aps.org/ supplemental/10.1103/PhysRevLett.110.066102 for AFM calibration details.

[20] F. J. Giessibl, Appl. Phys. Lett. 78, 123 (2001).

[21] J. E. Sader, J. Appl. Phys. 84, 64 (1998).

[22] Y. Zhu and S. Granick, Phys. Rev. Lett. 87, 096105 (2001).

[23] T. Becker and F. Mugele, Phys. Rev. Lett. 91, 166104 (2003).

[24] A. Labuda, Ph. D. thesis, McGill University, Canada, 2012, http://alekslabuda.com/sites/default/files/aleks_ labuda_phd_thesis.pdf.

[25] Y. Leng and P. T. Cummings, Phys. Rev. Lett. 94, 026101 (2005).

[26] M. Watkins and A.L. Shluger, Phys. Rev. Lett. 105, 196101 (2010).

[27] A. Malani and K. G. Ayappa, J. Phys. Chem. B 113, 1058 (2009).

[28] K. Kimura, S. Ido, N. Oyabu, K. Kobayashi, Y. Hirata, T. Imai, and H. Yamada, J. Chem. Phys. 132, 194705 (2010).

[29] A. Labuda, M. Lysy, and P. Grutter, Appl. Phys. Lett. 101, 113105 (2012). 